\title{
Normal Spanning Trees, Aronszajn Trees and Excluded Minors
}

\author{
Reinhard Diestel and Imre Leader
}

\begin{abstract}
We prove that a connected infinite graph has a normal spanning tree (the infinite analogue of a depth-first search tree) if and only if it has no minor obtained canonically from either an $\left(\aleph_{0}, \aleph_{1}\right)$-regular bipartite graph or an order-theoretic Aronszajn tree. This disproves Halin's conjecture that only the first of these obstructions was needed to characterize the graphs with normal spanning trees. As a corollary we deduce Halin's further conjecture that a connected graph has a normal spanning tree if and only if all its minors have countable colouring number.

The precise classification of the $\left(\aleph_{0}, \aleph_{1}\right)$-regular bipartite graphs remains an open problem. One such class turns out to contain obvious infinite minor-antichains, so as an unexpected corollary we reobtain Thomas's result that the infinite graphs are not well-quasi-ordered as minors.
\end{abstract}

\section{Introduction}

A spanning tree $T$ of a graph $G$ is called normal if the ends of every edge of $G$ are comparable in the natural tree order on $V(G)$ induced by $T$. Intuitively, all the edges of $G$ run vertically 'along' branches of $T$, never 'across'.

Normal spanning trees of finite graphs are more commonly known as depthfirst search trees. Every finite connected graph contains such a tree, and they are widely used as a structural tool in both algorithmic and pure graph theory.

Normal spanning trees of infinite graphs are just as useful; see e.g. $[6,7,8]$. However, not every infinite connected graph has a normal spanning tree: all countable ones do (see Jung's theorem below), but $K_{\aleph_{1}}$, say, does not. The purpose of this paper is to give a new characterization of the graphs containing normal spanning trees.

There is one well-known such characterization, due to Jung. Call a set $U$ of vertices of a graph $G$ dispersed in $G$ if every (1-way) infinite path in $G$ can be separated from $U$ by a finite set of vertices.

Theorem 1.1. (Jung 1969)

A connected graph $G$ has a normal spanning tree if and only if $V(G)$ is a countable union of dispersed sets.

However, Jung's result can be hard to make use of. For example, if a given graph has no normal spanning tree, then a characterization yielding some concrete 'forbidden' substructure in such cases would be more helpful than the abstract knowledge that the graph cannot be covered by countably many dispersed sets. Curiously, it was observed only recently by Halin [9] that, as 
an easy consequence of Jung's theorem, the class of graphs containing normal spanning trees can in principle be characterized by forbidden minors:

Corollary 1.2. Every connected minor of a graph admitting a normal spanning tree also has a normal spanning tree.

For completeness, we shall indicate in Section 3 how Corollary 1.2 follows from Jung's theorem. Interestingly, no direct proof appears to be known.

For an excluded-minor characterization of the graphs with normal spanning trees we would ideally try to compile a list (up to minor-equivalence) of all minor-minimal connected graphs not admitting a normal spanning tree, and prove that every other such graph contains one of these minimal ones as a minor. As things stand, however, we do not even know whether a minor-minimal graph without a normal spanning tree exists.

Yet there are 'types' of minors that seem to appear in every graph without a normal spanning tree. One such type is the following. Call a graph overloaded if it has uncountably many vertices each joined by infinitely many edges to some fixed countable set. It is not difficult to see that overloaded graphs cannot have normal spanning trees (see Section 3), and all known examples of graphs without normal spanning trees have overloaded minors.

Halin [9] conjectured that a connected graph has a normal spanning tree if and only if it has no overloaded minor. As we shall see, however, one needs another type of forbidden minor: there are connected graphs obtained from certain order-theoretic Aronszajn trees that have no normal spanning tree and no overloaded minor either. But these are all the obstructions needed: as our main result, we shall prove that every connected graph with no minor of either of these two types has a normal spanning tree.

We remark that we have not been able to classify either of the above two types of graphs up to minor-equivalence. In particular, although we shall present some minor-incomparable (and possibly minor-minimal) examples of overloaded graphs, we do not have a complete list of such graphs. This, or the related problem of classifying the $\left(\aleph_{0}, \aleph_{1}\right)$-regular bipartite graphs, seems to us to be a natural and interesting open problem.

Halin's conjecture would have implied that a connected graph has a normal spanning tree if and only if all its minors have countable colouring number. With some foresight, Halin [9] made this consequence a separate conjecture, and it will indeed follow easily from our main result.

Our paper is organized as follows. In Section 2 we briefly run through those of our terms that are not standard graph-theoretic terminology. In Section 3 we recall the contruction of normal spanning trees for countable connected graphs and look at some examples of graphs without a normal spanning tree; these will lead naturally to the formulation of our characterization theorem mentioned informally above. In Section 4 we review some tools from the theory of simplicial decompositions of infinite graphs that will be needed in the proof of our 
theorem; the proof itself is presented in Section 5. In Section 6 we construct a graph of Aronszajn-tree type that has no overloaded minor. Together with the fact that no (minimal) overloaded graph has a minor of Aronszajn-tree type, this shows that both types of excluded minor in our characterization are really needed. In Section 7 we prove Halin's second conjecture mentioned above. Our preliminary results towards a possible classification of overloaded graphs are presented in Section 8, where we exhibit two natural minor-incomparable examples of overloaded graphs. In Section 9, finally, we observe that one of these examples gives rise to a class of $2^{2^{\aleph_{0}}}$ minor-incomparable graphs of order $2^{\aleph_{0}}$ (assuming $\mathrm{CH}$ ).

\section{Definitions and basic facts}

We assume that the reader is familiar with standard graph-theoretic concepts and notation, eg. as defined in [4], but we assume no knowledge of set theory other than the most basic concepts (such as ordinal induction). We assume only the usual axioms of set theory (ZFC). For the construction of one of our examples in Section 8 we need the continuum hypothesis $(\mathrm{CH})$, and we shall say so at the time.

A ray is a 1-way infinite path. Two rays in a graph $G$ are equivalent if no finite set of vertices separates them in $G$.

When $G, H$ are graphs, we write $H \preccurlyeq G$ to express that $H$ is a minor of $G$. This means that with every vertex $x \in H$ we can associate a (possibly infinite) connected set $V_{x} \subseteq V(G)$, called the branch set of $x$, so that these sets $V_{x}$ are disjoint for different $x$ and $G$ contains a $V_{x}-V_{y}$ edge whenever $x y$ is an edge of $H$.

If $T$ is a (graph-theoretic) tree with root $r$, we write $x \leqslant y$ for vertices $x, y \in T$ if $x$ lies on the unique $r-y$ path in $T$. This is a partial ordering on $V(T)$; see [4] for a summary of some elementary properties.

A partially ordered set $(T, \leqslant)$ is called an order tree if all its subsets of the form $\lceil t\rceil=\lceil t\rceil_{T}:=\left\{t^{\prime} \mid t^{\prime} \leqslant t\right\}$ are well-ordered chains and $\lceil t\rceil \cap\left\lceil t^{\prime}\right\rceil \neq \emptyset$ for any $t, t^{\prime} \in T$. (Similarly, we write $\lfloor t\rfloor=\lfloor t\rfloor_{T}:=\left\{t^{\prime} \mid t^{\prime} \geqslant t\right\}$.) Note that an order tree has a unique minimal element, which is less than every other element. Our earlier partial ordering on the vertex set of a rooted graph-theoretic tree is an order tree in this sense.

Let $T$ be an order tree. A maximal chain in $T$ is called a branch of $T$; note that every branch inherits a well-ordering from $T$. The height of $T$ is the supremum of the order types of its branches. The height of a point $t \in T$ is the order type of $\lceil t\rceil^{\circ}:=\lceil t\rceil \backslash\{t\}$. The set $T^{\alpha}$ of all points at height $\alpha$ is the $\alpha$ th level of $T$, and we write $T^{<\alpha}:=\bigcup\left\{T^{\beta} \mid \beta<\alpha\right\}$.

The intuitive interpretation of a tree order as expressing height will also be used informally. For example, we may say that $t$ is above $t^{\prime}$ if $t>t^{\prime}$, call 
$\lceil X\rceil=\lceil X\rceil_{T}:=\bigcup\{\lceil x\rceil: x \in X\}$ the down-closure of $X \subseteq T$, or say that $X$ is down-closed if $X=\lceil X\rceil$. For a branch $B$ of $T$, we say that $X$ has unbounded $B$-height if $\lceil X\rceil \cap B \supseteq B$.

If $t<t^{\prime}$, we write $\left[t, t^{\prime}\right]$ for $\lfloor t\rfloor \cap\left\lceil t^{\prime}\right\rceil$, and call this set a (closed) interval in $T$. (Open and half-open intervals in $T$ are defined analogously.) A subset of $T$ that is an order tree under the ordering induced by $T$ is a subtree of $T$ if along with any two comparable points it contains the interval in $T$ between them. If $t<t^{\prime}$ but there is no point between $t$ and $t^{\prime}$, we call $t^{\prime}$ a successor of $t$ and $t$ the predecessor of $t^{\prime}$; if $t$ is not a successor of any point it is called a limit.

We say that an order tree $T$ is normal in a graph $G$ if $V(G)=T$ and the two ends of any edge of $G$ are comparable in $T$. We call $G$ a $T$-graph if $T$ is normal in $G$ and the set of lower neighbours of any point $t$ other than the root either consists of its predecessor (if $t$ is a successor in $T$ ) or else is a chain with supremum $t$ and of order type the cofinality of $\lceil t\rceil^{\circ}$. (In the $T$-graphs we shall consider, all limit points will have countable height, so their neighbourhoods will be $\omega$-chains.) Note that if $G$ is a $T$-graph then every interval $\left[t, t^{\prime}\right]$ in $T$ (and hence every subtree of $T$ ) is connected in $G$, because only $t$ can be a minimal element of any of its components.

A filter on a set $A$ is a non-empty set $\mathcal{F}$ of subsets of $A$ such that $\emptyset \neq \mathcal{F}$, any superset of an element of $\mathcal{F}$ is in $\mathcal{F}$, and $\mathcal{F}$ is closed under finite intersection. A maximal filter on $A$ is an ultrafilter on $A$. If $\mathcal{U}$ is an ultrafilter on $A$ and $A^{\prime} \subseteq A$, then exactly one of $A^{\prime}$ and $A \backslash A^{\prime}$ is an element of $\mathcal{U}$. A principal ultrafilter $\mathcal{U}$ on $A$ is one for which there exists an $a \in A$ such that $\mathcal{U}=\left\{A^{\prime} \subseteq A \mid a \in A^{\prime}\right\}$. Note that if $\mathcal{U}$ is a non-principal ultrafilter then for every finite subset of $A$ there is an element of $\mathcal{U}$ disjoint from it. Nonprincipal ultrafilters exist by Zorn's Lemma; indeed every maximal filter on $\mathbb{N}$ that extends the filter of all cofinite subsets of $\mathbb{N}$ is non-principal. See [2] for more details on ultrafilters.

Finally, we shall need the following simple version of Fodor's Lemma [10].

Lemma 2.1. If $f: \omega_{1} \rightarrow \omega_{1}$ is a function satisfying $f(\alpha)<\alpha$ for all non-zero $\alpha \in \omega_{1}$, then $f$ is constant on some uncountable subset of $\omega_{1}$.

Proof. Suppose not. Then for each $\alpha \in \omega_{1}$ there is a $\beta=\beta(\alpha)$ such that $f(\gamma) \geqslant \alpha$ for all $\gamma \geqslant \beta$. Now put $\alpha_{1}=1$ (say), and for $n=2,3, \ldots$ let $\alpha_{n}=\beta\left(\alpha_{n-1}\right)$. Then $\alpha:=\sup \alpha_{n}$ has $f(\alpha) \geq \alpha_{n}$ for all $n$, and hence $f(\alpha) \geqslant \alpha$; a contradiction. 


\section{Examples and statement of main result}

Our aim is to characterize the graphs that have a normal spanning tree. Before we look at various examples of graphs that contain no such tree, recall that, by Jung's Theorem, countable connected graphs do have normal spanning trees. We shall need a slight strengthening of this fact later, so let us indicate a direct proof.

Proposition 3.1. Let $G$ be a countable connected graph, and let $v_{1}, \ldots, v_{n}$ be a finite path in $G$. Then $G$ has a normal spanning tree with root $v_{1}$ in which $v_{i+1}$ is a successor of $v_{i}$ for all $i=1, \ldots, n-1$.

Proof. Let $v_{1}, v_{2}, \ldots$ be an enumeration of $V(G)$. Let $T_{1}$ be the path $v_{1} \ldots v_{n}$. Assume inductively that, for some $i \in \mathbb{N}$, we have constructed a finite tree $T_{i} \subseteq G$ with root $v_{1}$ that contains $v_{1}, \ldots, v_{i}$ and has the property that for every component $C$ of $G-T_{i}$ the set $N(C)$ of neighbours of $C$ on $T_{i}$ is a chain in $T_{i}$. Let $j$ be minimal such that $v_{j} \notin T_{i}$, let $C$ be the component of $G-T_{i}$ containing $v_{j}$, and let $t$ be the maximum of the chain $N(C)$ in $T_{i}$. Let $P=t \ldots v_{j}$ be a path whose only vertex in $T_{i}$ is $t$, and put $T_{j}:=T_{i} \cup P$. As is easily checked, the (nested) union of all the trees $T_{i}$ constructed in this way is a normal spanning tree of $G$ with root $v_{1}$.

For completeness, we now indicate how Jung's theorem implies that connected minors of graphs with normal spanning trees again have a normal spanning tree. The reader is encouraged to skip the proof, which is no more than a straightforward application of the definitions involved.

Proof of Corollary 1.2 (Sketch). If $H$ is a minor of $G$ and $U \subseteq V(G)$ is a dispersed set of vertices in $G$, then the set $W$ of all vertices of $H$ whose branch set meets $U$ is dispersed in $H$ : any ray $R \subseteq H$ has a ray of $G$ contained in the union of the branch sets of its vertices, and if this latter ray in $G$ is separated from $U$ by the finite set $S$, then the finite set of vertices of $H$ whose branch set meets $S$ separates $R$ from $W$ in $H$. Hence if $G$ is a countable union of dispersed sets then so is $H$, and the assertion follows from Theorem 1.1.

Let us now look at some connected graphs that have no normal spanning tree. The prime example is, of course, the complete graph $K_{\aleph_{1}}$ : since every two vertices would have to be comparable, any order tree normal in $K_{\aleph_{1}}$ would consist of a single chain and could not be a graph-theoretic tree.

For more subtle examples, we need the following two easily verified properties of normal trees. (See [1] or [8] for proofs.)

Lemma 3.2. Let $T$ be an order tree that is normal in a graph $G$, and let $x, y \in T$.

(i) If $x, y$ are incomparable in $T$, then $\lceil x\rceil^{\circ} \cap\lceil y\rceil^{\circ}$ separates $x$ from $y$ in $G$.

(ii) Every ray in $G$ meets some branch of $T$ infinitely often. 
Now if $T$ is a normal spanning tree of $G$, then by (i) any two branches of $T$ are separated in $G$ by their finite intersection. Hence two rays whose branches in (ii) differ cannot be equivalent: they would have tails in different components of $G$ minus the finite intersection of those branches. Thus, no graph with a normal spanning tree can have uncountably many disjoint equivalent rays, as these would all meet the same countable branch.

Our first non-trivial example of a connected graph with no normal spanning tree, then, is the cartesian product of an uncountable star with a raya graph that certainly contains uncountably many disjoint equivalent rays. (In fact, it is easy to check that any two rays in this graph are equivalent.)

However, the above example can easily be simplified: just contract each copy of the original ray that corresponds to a leaf of the star, and delete the edges of the central ray. The graph $G$ obtained is a $K_{\aleph_{0}, \aleph_{1}}$, and this, too, has no normal spanning tree. Indeed, any such tree $T$ has only countably many levels, and hence has a level $T^{n}$ containing uncountably many vertices from the uncountable class $B$ of $G$. Each of these vertices $b$ has infinitely neighbours in the countable class $A$, but at most $n$ of these can lie below it. So every $b$ has neighbour $a$ above it. As the $b$ are pairwise incomparable, their upper neighbours $a$ are pairwise distinct, which contradicts the fact that $A$ is countable.

Note that in the above proof the only properties of $G$ used were the sizes of $A$ and $B$ and the degrees of the vertices in $B$. Let us call an arbitrary bipartite graph $(A, B)$ an $\left(\aleph_{0}, \aleph_{1}\right)$-graph if $|A|=\aleph_{0}$ and $|B|=\aleph_{1}$ and every vertex in $B$ has infinite degree. (Thus, a graph is overloaded if and only if it contains an $\left(\aleph_{0}, \aleph_{1}\right)$-graph as a subgraph.) We remark in passing that deleting all the vertices of countable degree from $A$ and all their neighbours from $B$ yields another $\left(\aleph_{0}, \aleph_{1}\right)$-graph in which every vertex in $A$ has uncountable degree.

We have thus reobtained Halin's [9] observation:

Proposition 3.3. No overloaded graph has a normal spanning tree.

The following observation shows that, as a 'type' of graphs, the $\left(\aleph_{0}, \aleph_{1}\right)$ graphs are minimal among the graphs not containing normal spanning trees.

Proposition 3.4. Every connected minor of an $\left(\aleph_{0}, \aleph_{1}\right)$-graph is either itself overloaded or contains a normal spanning tree.

Proof. Let $G=(A, B)$ be an $\left(\aleph_{0}, \aleph_{1}\right)$-graph, and let $H \preccurlyeq G$ be connected. Let $A^{\prime}$ denote the set of vertices of $H$ whose branch set meets $A$, let $B^{\prime}$ be the set of those vertices of $H-A^{\prime}$ that have infinitely many neighbours in $A^{\prime}$, and let $B^{\prime \prime}:=V(H) \backslash\left(A^{\prime} \cup B^{\prime}\right)$. Note that $B^{\prime} \cup B^{\prime \prime}$ is an independent set in $H$, with singleton branch sets in $B$. If $B^{\prime}$ is uncountable, then $\left(A^{\prime}, B^{\prime}\right)$ is an $\left(\aleph_{0}, \aleph_{1}\right)$-subgraph of $H$, so $H$ is overloaded.

So let us assume that $B^{\prime}$ is countable. For every finite set $F \subseteq A^{\prime}$ let $B^{\prime \prime}(F)$ consist of those points in $B^{\prime \prime}$ whose neighbourhood is precisely $F$. Since 
$A^{\prime}$ is countable, there are only countably many such sets $B^{\prime \prime}(F)$, and together with the singleton sets consisting of elements of $A^{\prime} \cup B^{\prime}$ they form a partition of $V(H)$ into countably many dispersed sets. By Jung's theorem, $H$ thus has a normal spanning tree.

In Section 8 , we shall study $\left(\aleph_{0}, \aleph_{1}\right)$-graphs more closely; they will turn out to show some perhaps unexpected degree of variety. As for now, let us mention just one natural example of an $\left(\aleph_{0}, \aleph_{1}\right)$-graph that looks essentially different from a $K_{\aleph_{0}, \aleph_{1}}$. Let $T_{2}$ be the order tree of height $\omega+1$ in which every point of finite height has exactly two successors and above every branch of $T_{2}^{<\omega}$ there is exactly one point in $T_{2}^{\omega}$. We shall call the down-closure of any uncountable subset $X$ of $T_{2}^{\omega}$ a binary tree with tops, the tops themselves being the points in $X$. Now if $T$ is a binary tree with $\aleph_{1}$ tops then any $T$-graph clearly contains an $\left(\aleph_{0}, \aleph_{1}\right)$-graph with vertex classes $T^{<\omega}$ and $T^{\omega}$; we shall call any such graph an $\left(\aleph_{0}, \aleph_{1}\right)$-graph of binary type.

Are there any connected graphs without a normal spanning tree that have no overloaded minor? Good candidates for such graphs might seem to be the $\omega_{1}$-graphs (where $\omega_{1}$ is viewed as an order tree). Indeed in any normal spanning tree of an uncountable graph $G$ there is a point $t$ with uncountably many successors, and by Lemma 3.2 the finite set $\lceil t\rceil$ separates these pairwise in $G$. But if $G$ is an $\omega_{1}$-graph then a finite separator can leave only finitely many components, because intervals in $\omega_{1}$-graphs are connected.

So an $\omega_{1}$-graph cannot have a normal spanning tree. And neither can it itself be overloaded, i.e. have an $\left(\aleph_{0}, \aleph_{1}\right)$-subgraph $(A, B)$ : since $A$ is countable, all but countably many of the vertices in $B$ would lie above $\alpha:=\sup A$, and vertices above $\alpha$ have only finitely many neighbours below it. (Recall the definition of a $T$-graph.)

However, $\omega_{1}$-graphs do contain overloaded minors, and far more:

Proposition 3.5. Every $\omega_{1}$-graph $G$ has a $K_{\aleph_{1}}$-minor.

Proof. Note first that $G$ has uncountably many vertices $v_{\alpha}\left(\alpha<\omega_{1}\right)$ of uncountable degree: these can be found inductively by applying Lemma 2.1 (our weak version of Fodor's Lemma) to final segments of $\omega_{1}$, using the fact that every vertex above a point $\alpha<\omega_{1}$ has a lower neighbour above or equal to $\alpha$. Using (some of) the vertices $v_{\alpha}$ as branch vertices, we can now build a topological $K_{\aleph_{1}}$ minor of $G$ inductively in $\omega_{1}$ steps, at each step adding a new branch vertex $v_{\alpha}$ and joining it to the previously selected $v_{\beta}$ one by one, linking a sufficiently high neighbour $x$ of $v_{\beta}$ to a sufficiently high neighbour $y$ of $v_{\alpha}$ by a path in $[x, y]$.

Thus if we are looking for a $T$-graph $G$ that has no overloaded minor, then $T$ must not contain uncountable chains. If we assume further that $T$ has no point with uncountably many successors (an assumption clearly desirable since 
we wish to keep $G$ 'small'-ideally, minor-minimal without a normal spanning tree), it follows that every level of $T$ must be countable: if $T^{\alpha}$ were the first uncountable level, then $\alpha$ would be a limit ordinal, and $G$ would contain an $\left(\aleph_{0}, \aleph_{1}\right)$-graph with vertex sets $T^{<\alpha}$ and $T^{\alpha}$.

So we are looking for $T$-graphs where $T$ is an uncountable order tree whose branches and levels are all countable. Such trees are called Aronszajn trees, and they are not entirely trivial to construct; the first one was found by Aronszajn, see [14]. Note that all Aronszajn trees have height $\omega_{1}$ and order $\aleph_{1}$. We refer the reader to Jech [10] for general background.

Despite the two restrictions on $T$, such $T$-graphs are still too large to have normal spanning trees:

Proposition 3.6. Let $T$ be an Aronszajn tree, and let $G$ be a $T$-graph. Then $G$ has no normal spanning tree.

Proof. If $G$ has a normal spanning tree, then some point $t$ in it has uncountably many successors, and $G-\lceil t\rceil$ has uncountably many components (Lemma 3.2). Let $\alpha<\omega_{1}$ be such that $\lceil t\rceil \subseteq T^{<\alpha}$. Since $T$ is an Aronszajn tree, both $T^{<\alpha}$ and $T^{\alpha}$ are countable. Since $G$ is a $T$-graph, any component of $G-\lceil t\rceil$ not meeting $T^{<\alpha}$ has the form $\left\lfloor t^{\prime}\right\rfloor$ for some $t^{\prime} \in T^{\alpha}$. So $G-\lceil t\rceil$ has only countably many components, a contradiction.

Like $\omega_{1}$-graphs, Aronszajn-tree graphs (that is, $T$-graphs with $T$ an Aronszajn tree) cannot themselves be overloaded. But can they have overloaded minors?

Some certainly do. Indeed, let $T$ be any Aronszajn tree, and let $G$ be any $T$-graph. Let $t \in T^{\omega}$ be such that $\lfloor t\rfloor$ is uncountable, and let $a_{1}<a_{2}<\ldots$ be the points below $t$. We shall add some edges to $G$ in such a way that $G$ remains a $T$-graph but acquires a $K_{\aleph_{0}, \aleph_{1}}$ minor $(A, B)$ for which $\left\{a_{1}\right\},\left\{a_{2}\right\}, \ldots$ are the branch sets of the vertices in $A$. Let us construct the (countable) branch sets $X_{\alpha} \subseteq T$ of the vertices $b_{\alpha} \in B$ inductively for all $\alpha<\omega_{1}$, and add edges from $X_{\alpha}$ to all the $a_{i}$ at the same time. Let $\alpha<\omega_{1}$ be given, and assume that $X_{\beta}$ has been defined for every $\beta<\alpha$. Let $\gamma<\omega_{1}$ be large enough that $X_{\beta} \subseteq T^{<\gamma}$ for every $\beta<\alpha$, and pick a point $s>t$ at level $\gamma$. Then pick distinct limits $t_{1}, t_{2}, \ldots$ above $s$, add all the edges $t_{i} a_{i}(i \in \mathbb{N})$, and let

$$
X_{\alpha}:=\lfloor s\rfloor \cap \bigcup\left\{\left\lceil t_{i}\right\rceil: i \in \mathbb{N}\right\} .
$$

Note that $X_{\alpha}$ is connected in $G$, that at most one down-edge is added at each limit in $T$, and no down-edges are added at successors. So when the construction is complete we have $K_{\aleph_{0}, \aleph_{1}} \preccurlyeq G$, and $G$ is still a $T$-graph.

In general, however, it turns out that Aronszajn-tree graphs need not have overloaded minors. As our second main result, we shall construct such an Aronszajn-tree graph in Section 6. 
Conversely, it is easy to see that $\left(\aleph_{0}, \aleph_{1}\right)$-graphs do not contain Aronszajntree graphs as minors (Proposition 6.1). So we have found two types of connected graphs that have no normal spanning trees and do not, in general, have a minor of the other type: $\left(\aleph_{0}, \aleph_{1}\right)$-graphs and Aronszajn-tree graphs. Our main result is that these are all we need in order to characterize the graphs without a normal spanning tree:

Theorem 3.7. A connected graph has a normal spanning tree if and only if it contains neither an $\left(\aleph_{0}, \aleph_{1}\right)$-graph nor an Aronszajn-tree graph as a minor.

We shall prove this theorem in the following two sections.

\section{Simplicial decompositions}

Let $G$ be a graph, $\sigma>0$ an ordinal, and for each $\lambda<\sigma$ let $B_{\lambda}$ be an induced subgraph of $G$. The family $F=\left(B_{\lambda}\right)_{\lambda<\sigma}$ is called a simplicial decomposition of $G$ if the following three conditions hold:

(S1) $G=\bigcup_{\lambda<\sigma} B_{\lambda}$;

(S2) every $S_{\mu}:=B_{\mu} \cap \bigcup_{\lambda<\mu} B_{\lambda}$ is a complete graph $\quad(0<\mu<\sigma)$;

(S3) no $S_{\mu}$ contains $B_{\mu}$ or any other $B_{\lambda} \quad(0 \leqslant \lambda<\mu<\sigma)$.

The subgraphs $B_{\lambda}$ are the parts of this decomposition.

For every vertex $v \in G$ we let $\lambda(v)$ denote the minimal $\lambda$ for which $v \in B_{\lambda}$. For $X \subseteq V(G)$ we put $\Lambda(X):=\{\lambda(x) \mid x \in X\}$. Thus, the vertices $x$ with $\lambda(x)=\mu$ are precisely those in $B_{\mu} \backslash S_{\mu} . F$ will be called coherent if, for each $\mu<\sigma, B_{\mu} \backslash S_{\mu}$ is connected, $S_{\mu} \neq \emptyset$ if $\mu>0$, and every vertex of $S_{\mu}$ has a neighbour in $B_{\mu} \backslash S_{\mu}$.

The decomposition tree $\left(T_{F}, \leqslant\right)$ associated with $F$ is the order tree on $F$ defined recursively as follows. Let $\mu<\sigma$ be given, and assume that $\leqslant$ has been defined for all pairs $\left(B_{\lambda}, B_{\lambda^{\prime}}\right)$ with $\lambda, \lambda^{\prime}<\mu$. For each $\lambda<\mu$, let

$$
B_{\lambda}<B_{\mu} \quad \text { if } B_{\lambda} \leqslant B_{\lambda(s)} \text { for some } s \in S_{\mu} ;
$$

otherwise let $B_{\lambda}$ and $B_{\mu}$ be incomparable. Note that $T_{F}$ has height at most $\sigma$, and that $\left\{B_{\lambda} \mid \lambda \in \Lambda\left(S_{\mu}\right)\right\}$ is a cofinal subchain of the chain $\left\lceil B_{\mu}\right\rceil^{\circ}$ in $T_{F}$ below the point $B_{\mu}$. See [3] for details about simplicial decompositions, including a (straightforward) proof that $T_{F}$ is indeed an order tree.

The following lemma is easily proved by induction on $\sigma$.

Lemma 4.1. For every $\mu<\sigma$, the subgraph $S_{\mu}$ separates $\bigcup\left\lfloor B_{\mu}\right\rfloor_{T_{F}}$ from the rest of $G$.

The main tool in our proof of Theorem 3.7 will be the following result from [3; Ch. 5, Thm. 2.1]: 


\section{Theorem 4.2. (Halin 1967)}

Let $G$ be a connected graph in which two vertices are adjacent whenever they are linked by uncountably many independent paths. If $K_{\aleph_{1}} \nsubseteq G$ then $G$ admits a coherent simplicial decomposition $\left(B_{\lambda}\right)_{\lambda<\sigma}$ into countable parts, with $\sigma \leqslant \omega_{1}$.

The seemingly narrow and technical adjacency condition in Halin's theorem will not pose a major obstacle when we come to use the result in our proof of Theorem 3.7, due to the following easy lemma (proved in [3]). Given a graph $G$, let $[G]_{\aleph_{1}}$ denote the supergraph of $G$ obtained by joining any nonadjacent vertices $x, y$ for which $G$ contains uncountably many $x-y$ paths.

Lemma 4.3. $\left[[G]_{\aleph_{1}}\right]_{\aleph_{1}}=[G]_{\aleph_{1}}$

Thus even if our given graph $G$ falls short of the adjacency condition, the graph $[G]_{\aleph_{1}}$ will satisfy it. Our next lemma says that the coherence of the simplicial decomposition of $[G]_{\aleph_{1}}$ given by Halin's theorem may be assumed to rely only on edges of $G$ :

Lemma 4.4. Let $G$ be a graph such that $G^{\prime}:=[G]_{\aleph_{1}}$ has a coherent simplicial decomposition $F=\left(B_{\lambda}\right)_{\lambda<\sigma}$ into countable parts, where $\sigma \leqslant \omega_{1}$. Then $G^{\prime}$ has a simplicial decomposition $\left(B_{\lambda}^{*}\right)_{\lambda \in \Lambda \subseteq \sigma}$ into countable parts such that the following assertions hold for every $\mu \in \Lambda$ :

(i) $S_{\mu}^{*}=S_{\mu} \quad\left(\right.$ where $\left.S_{\mu}^{*}:=B_{\mu}^{*} \cap \bigcup_{\lambda \in \Lambda \cap \mu} B_{\lambda}^{*}\right)$;

(ii) $V\left(B_{\mu}^{*} \backslash S_{\mu}^{*}\right)$ is connected in $G$;

(iii) every vertex in $S_{\mu}^{*}$ sends an edge of $G$ to $B_{\mu}^{*} \backslash S_{\mu}^{*}$.

Proof. Let us define the graphs $B_{\lambda}^{*}$ and their index set $\Lambda \subseteq \sigma$ recursively. Along with every $\lambda \in \Lambda$, we shall select a countable subtree $T_{\lambda}$ of $T_{F}$ and put

$$
B_{\lambda}^{*}:=\bigcup T_{\lambda}
$$

At each time of the recursion, the union $T$ of the subtrees selected so far will be down-closed in $T_{F}$.

Let $\Lambda^{\prime}$ be the subset of $\Lambda$ selected so far, and put

$$
T:=\bigcup_{\lambda \in \Lambda^{\prime}} T_{\lambda}
$$

If $T \neq T_{F}$, let $B_{\mu}$ be a minimal point of $T_{F} \backslash T$. As $T$ is down-closed in $T_{F}$, Lemma 4.1 implies that $\bigcup\left\lfloor B_{\mu}\right\rfloor$ meets $\bigcup T=\bigcup_{\lambda \in \Lambda^{\prime}} B_{\lambda}^{*}$ only in $S_{\mu}$. (This will ensure assertion (i) of the lemma.) We select $\mu$ for membership in $\Lambda$, and will now construct $T_{\mu}$.

We first seek to ensure that $B_{\mu}^{*}$ will satisfy assertion (iii), assuming (i). Since $F$ is coherent, every vertex $s \in S_{\mu}$ sends an edge of $G^{\prime}$ to some vertex $v \in B_{\mu} \backslash S_{\mu}$. If $s v$ is an edge of $G$, put $v=: x(s)$. If not, then $G$ contains 
uncountably many $s-v$ paths. Only countably many of these meet $S_{\mu}-s$; let $x(s)$ be a neighbour of $s$ on one of the other paths. Then

$$
X:=\left\{x(s) \mid s \in S_{\mu}\right\}
$$

is a countable subset of $\bigcup\left\lfloor B_{\mu}\right\rfloor \backslash S_{\mu}$, and we put

$$
\left.T_{\mu}^{0}:=\left\lceil\left\{B_{\lambda} \mid \lambda \in \Lambda(X)\right\}\right\rceil \backslash B_{\mu}\right\rceil^{\circ}
$$

Note that $T_{\mu}^{0}$ is countable, because $X$ is countable and $T_{F}$ has height at most $\omega_{1}$ (so each $B_{\lambda}$ has countable height). For every $G^{\prime}$-edge $x y$ of the (countable) graph $\left(\bigcup T_{\mu}^{0}\right) \backslash S_{\mu}$ that is not an edge of $G$ we now similarly select an $x-y$ path $P \subseteq G$ that avoids $S_{\mu}$, let $H_{1}$ denote the union of all these paths, and put

$$
T_{\mu}^{1}:=\left\lceil T_{\mu}^{0} \cup\left\{B_{\lambda} \mid \lambda \in \Lambda\left(H_{1}\right)\right\}\right\rceil \backslash\left\lceil B_{\mu}\right\rceil^{\circ} .
$$

We now repeat this last extension $\omega$ times, at each step adding an $x-y$ path in $G$ for each of the (countably many) $G^{\prime}$-edges of the graph $\left(\bigcup T_{\mu}^{i}\right) \backslash S_{\mu}$ constructed so far, and then adding the down-closure (above $B_{\mu}$ ) of all the parts of $F$ needed to accommodate these paths. Then

$$
T_{\mu}:=\bigcup_{i \in \mathbb{N}} T_{\mu}^{i}
$$

is still countable, and $B_{\mu}^{*}=\bigcup T_{\mu}$ satisfies assertion (ii) of the lemma. The other assertions are easily checked.

To wind up the induction step of the construction, note that as $B_{\mu}$ was minimal in $T_{F} \backslash T$ and each $T_{\mu}^{i}$ was down-closed in $\left\lfloor B_{\mu}\right\rfloor$, the union of $T$ and $T_{\mu}$ is again down-closed in $T_{F}$. And since nested unions of down-closed subtrees are again down-closed, the tree $\bigcup_{\lambda \in \Lambda^{\prime}} T_{\lambda}$ will be down-closed in $T_{F}$ also at limit steps of the construction.

Lemma 4.5. Let $F=\left(B_{\lambda}\right)_{\lambda<\sigma}$ be a coherent simplicial decomposition of a graph $G^{\prime}$, where $\sigma \leqslant \omega_{1}$. Let $G$ be a spanning subgraph of $G^{\prime}$ such that $V\left(B_{\mu} \backslash S_{\mu}\right)$ is connected in $G$ and every vertex in $S_{\mu}$ sends an edge of $G$ to $B_{\mu} \backslash S_{\mu}$, for all $\mu<\sigma$. Let $H \preccurlyeq G$ be the minor of $G$ obtained by contracting each of the subgraphs $G \cap\left(B_{\mu} \backslash S_{\mu}\right)$ to a vertex $x_{\mu}$. Then $H$ contains a $T_{F^{-}}$ graph.

Proof. To define a $T_{F}$-graph $H^{\prime}$ on $T_{F}$, we need to specify for each $B_{\mu} \in T_{F}$ the parts $B_{\lambda}$ below $B_{\mu}$ to which $B_{\mu}$ is to send an edge of $H^{\prime}$. If $\Lambda\left(S_{\mu}\right)$ has a maximal element $\lambda$, then $B_{\mu}$ is a successor of $B_{\lambda}$ in $T_{F}$ and we make $B_{\mu}$ adjacent just to $B_{\lambda}$. If not, choose $\Lambda \subseteq \Lambda\left(S_{\mu}\right)$ so that $\left\{B_{\lambda} \mid \lambda \in \Lambda\right\}$ is an $\omega$-chain cofinal in $\left\lceil B_{\mu}\right\rceil^{\circ}$ (this is possible because $\left\{B_{\lambda} \mid \lambda \in \Lambda\left(S_{\mu}\right)\right\}$ is cofinal in $\left\lceil B_{\mu}\right\rceil^{\circ}$ and $T_{F}$ has height at most $\sigma \leqslant \omega_{1}$ ), and make $B_{\mu}$ adjacent to every $B_{\lambda}$ with $\lambda \in \Lambda$. 
This graph $H^{\prime}$ clearly is a $T_{F}$-graph. Moreover, for every edge $B_{\lambda} B_{\mu}$ in $H^{\prime}$ (with $\lambda<\mu$ ) there is a vertex $s \in S_{\mu}$ with $\lambda(s)=\lambda$ and hence $s \in B_{\lambda} \backslash S_{\lambda}$. By assumption, $s$ sends an edge of $G$ to $B_{\mu} \backslash S_{\mu}$. The map $B_{\mu} \mapsto x_{\mu}$ thus is a subgraph embedding of $H^{\prime}$ in $H$.

\section{Proof of the main result}

The forward implication of Theorem 3.7 has already been established in Corollary 1.2 and Propositions 3.3 and 3.6. For the converse implication, let $G$ be a connected graph, and put $G^{\prime}:=[G]_{\aleph_{1}}$. We shall either find an $\left(\aleph_{0}, \aleph_{1}\right)$-graph or Aronszajn-tree minor in $G$, or succeed in constructing a normal spanning tree of $G^{\prime}$; by Corollary 1.2, there will then also be a normal spanning tree in $G$.

Since $K_{\aleph_{1}}$ contains an $\left(\aleph_{0}, \aleph_{1}\right)$-graph, we may assume that $G$ has no $K_{\aleph_{1}}$ minor. Then $G^{\prime}$ has no $K_{\aleph_{1}}$ subgraph: if it did, we could turn it into a $T K_{\aleph_{1}} \subseteq G$ by selecting branch vertices inductively in $\omega_{1}$ steps and replacing missing edges from a new branch vertex to the countably many previously selected branch vertices by paths in $G$ avoiding the (countable) part of the $T K_{\aleph_{1}}$ constructed so far. By Lemma 4.3 we may thus apply Theorem 4.2 to $G^{\prime}$, obtaining a coherent simplicial decomposition $\left(B_{\lambda}\right)_{\lambda<\sigma}$ of $G^{\prime}$ into countable parts, with $\sigma \leqslant \omega_{1}$. Let $F=\left(B_{\lambda}^{*}\right)_{\lambda \in \Lambda}$ be the simplicial decomposition of $G^{\prime}$ obtained from this decomposition as in Lemma 4.4.

For every subtree $T$ of $T_{F}$, we may apply Lemma 4.5 to the simplicial decomposition of $\bigcup T$ induced by $F$ to obtain a $T$-graph as a minor of $G$. We may therefore assume that no subtree of $T_{F}$ is an Aronszajn tree.

Consider the down-closed subtree

$$
T_{0}:=\left\{B^{*} \mid \forall \mu \in \Lambda:\left(\min T_{F}<B_{\mu}^{*} \leqslant B^{*} \Rightarrow\left|S_{\mu}^{*}\right|=\aleph_{0}\right)\right\}
$$

of $T_{F}$. Suppose $T_{0}$ is uncountable. By Proposition 3.5 and Lemma 4.5, and our assumption that $G$ has no $K_{\aleph_{1}}$ minor, $T_{0}$ has no uncountable chain. Since $T_{0}$ is not an Aronszajn tree, it thus has an uncountable level $T_{0}^{\alpha}$. If $\alpha$ is chosen minimal, then $A:=V\left(\bigcup T_{0}^{<\alpha}\right)$ is countable. For each of the uncountably many $B_{\mu}^{*} \in T_{0}^{\alpha}$, every vertex in $S_{\mu}^{*}$ sends an edge of $G$ to $B_{\mu}^{*} \backslash S_{\mu}^{*}$. By definition of $T_{0}$, these $S_{\mu}^{*}$ are infinite, and by assumption every set $V\left(B_{\mu}^{*} \backslash S_{\mu}^{*}\right)$ is connected in $G$. Contract each of these connected sets to a vertex $b_{\mu}$, and set

$$
B:=\left\{b_{\mu} \mid B_{\mu}^{*} \in T_{0}^{\alpha}\right\}
$$

to obtain an $\left(\aleph_{0}, \aleph_{1}\right)$-minor $(A, B)$ of $G$.

We may thus assume that $T_{0}$ is countable. Since $F$ is coherent,

$$
G_{0}^{\prime}:=\bigcup T_{0}
$$


is connected and thus has a normal spanning tree $T_{0}^{\prime}$ by Proposition 3.1.

We now delete $T_{0}$ from $T_{F}$ and apply the same construction to any of the maximal subtrees $T$ of $T_{F} \backslash T_{0}$. (There is one such tree $T=\left\lfloor B^{*}\right\rfloor$ for each minimal element $B^{*}$ of $T_{F} \backslash T_{0}$.) Indeed, let

$$
T_{1}:=\left\{B^{*} \mid \forall \mu \in \Lambda:\left(\min T<B_{\mu}^{*} \leqslant B^{*} \Rightarrow\left|S_{\mu}^{*}\right|=\aleph_{0}\right)\right\} .
$$

As for $T_{0}$, we may assume that $T_{1}$ is countable, and use Proposition 3.1 to find a normal spanning tree $T_{1}^{\prime}$ in

$$
G_{1}^{\prime}:=\bigcup T_{1}
$$

In order to make $T_{1}^{\prime}$ compatible with $T_{0}^{\prime}$, we apply Proposition 3.1 with the vertices $v_{1}, \ldots, v_{n}$ of $S_{\mu}^{*}$ for $B_{\mu}^{*}:=\min T$, listed in their ascending order in $T_{0}^{\prime}$. ( $S_{\mu}^{*}$ is finite because $B_{\mu}^{*}$ is minimal in $T_{F} \backslash T_{0}$, and $v_{1}, \ldots, v_{n}$ form a chain in $T_{0}^{\prime}$ because they span a complete graph in $G_{0}^{\prime}$.) If $T_{1}^{\prime}$ is chosen in this way, then $T_{0}^{\prime} \cup T_{1}^{\prime}$ is a normal spanning tree of $G_{0}^{\prime} \cup G_{1}^{\prime}$.

Repeating this step transfinitely until $T_{F}$ is exhausted, we obtain a nested sequence of normal spanning trees $\bigcup_{\beta<\alpha} T_{\beta}^{\prime}$ of induced subgraphs $\bigcup_{\beta<\alpha} G_{\beta}^{\prime}$ of $G^{\prime}$, whose union is clearly a normal spanning tree of $G^{\prime}$.

\section{Aronszajn-tree graphs without overloaded minors}

Our aim in this section is to show that both types of the minors used in Theorem 3.7 are actually needed: that there are Aronszajn-tree graphs without overloaded minors, and vice versa. The latter of these is immediate:

Proposition 6.1. No $\left(\aleph_{0}, \aleph_{1}\right)$-graph has an Aronszajn-tree minor.

Proof. Let $H$ be a $T$-graph with $T$ an Aronszajn tree, and suppose that $H$ is a minor of an $\left(\aleph_{0}, \aleph_{1}\right)$-graph $(A, B)$. Only countably many vertices of $H$ have branch sets that meet $A$; let $\alpha<\omega_{1}$ be large enough that all these vertices lie in $T^{<\alpha}$. The uncountably many vertices of $H$ that are not in $T^{<\alpha}$ then are singleton elements of $B$. So they form an independent set in $H$, and must hence all lie in $T^{\alpha}$. As Aronszajn trees have no uncountable levels, this is a contradiction.

We now turn our attention to the main result of this section, the construction of an Aronszajn-tree graph $G$ that has no overloaded minor. As a first attempt, one might try to pick an arbitrary Aronszajn tree $T$ and try to choose the edges for $G$ inductively to avoid creating an overloaded minor. However, our attempts in this direction all led to overloaded minors arising similarly to 
Click here for a direct construction of an Aronszajn tree $T$ and a graph on $T$ that has no overloaded minor.

those in $\omega_{1}$-graphs. The crucial idea here seems to be to find instead some 'global' condition on $G$ (such as condition $(*)$ in the proof below) that prevents overloaded minors, although this is not necessarily easy to achieve by choosing the edges inductively (i.e., with reference only to points below the point whose incident edges are being defined).

An Aronszajn tree is called regular or special if it has a partition into countably many antichains. The standard construction of an Aronszajn tree (see [10]) yields such a 'special' tree.

Theorem 6.2. On every special Aronszajn tree $T$ there exists a $T$-graph that has no overloaded minor.

Proof. Let $T$ be a special Aronszajn tree, with an antichain partition $\left(U_{n}\right)_{n \in \omega}$ say. We shall construct a $T$-graph $G$ on $T$ with the following property:

For every $t \in T$ there is a finite set $S_{t} \subseteq\lceil t\rceil^{\circ}$ such that every $t^{\prime}>t$ has all its neighbours below $t$ inside $S_{t}$.

Let us see first why no such graph $G$ can have an $\left(\aleph_{0}, \aleph_{1}\right)$-minor $(A, B)$. Suppose it does. For each $v \in(A, B)$ let $t_{v}$ be the minimal point in $T$ of the branch set of $v$; this point is unique by Lemma 3.2 (i), because the branch set is connected. Since $A$ is countable, there exists an $\alpha<\omega_{1}$ such that $t_{a} \in T^{<\alpha}$ for every $a \in A$. Pick $b \in B$ with $t_{b}$ of height $\geqslant \alpha+\omega$; such $b$ exists, since $T^{<\alpha+\omega}$ is countable but $B$ is uncountable.

Only finitely many neighbours of $b$ have branch sets meeting $S_{t_{b}}$; let $A_{1}, A_{2}, \ldots$ be the branch sets of its other neighbours. None of these connected sets $A_{i}$ meets $\left\lfloor t_{b}\right\rfloor$ : if one did, it would contain an edge between $\left\lfloor t_{b}\right\rfloor \backslash\left\{t_{b}\right\}$ and $T \backslash\left\lfloor t_{b}\right\rfloor$ (where its minimum lies), and any such edge would be incident with a vertex in $S_{t_{b}}$. For the same reason, the only vertex in $\left\lfloor t_{b}\right\rfloor$ that can have a neighbour in any of the $A_{i}$ is $t_{b}$. Since the branch set of $b$ sends an edge to every $A_{i}$ and lies in $\left\lfloor t_{b}\right\rfloor$, this means that $t_{b}$ has a neighbour in every $A_{i}$. As only finitely many lower neighbours of $t_{b}$ have height $\leqslant \alpha$ (recall the definition of a $T$-graph), infinitely many $A_{i}$ meet the interval $\left(t, t_{b}\right)$, where $t$ is the point in $\left\lceil t_{b}\right\rceil^{\circ}$ at height $\alpha$. But every $A_{i}$ has its minimum outside $\lfloor t\rfloor$, so infinitely many $A_{i}$ have an edge between a point $t^{\prime}>t$ and a point below $t$. As these edges are independent, this contradicts $(*)$.

It remains to show that we can indeed choose the edges of $G$ so as to satisfy $(*)$. Given a limit point $t \in T$, let us choose its neighbours $t_{1}<t_{2} \ldots$ below $t$ inductively, starting with an arbitrary point $t_{1}<t$. Now let $n>1$ be given, and suppose that $t_{1}<\ldots<t_{n-1}<t$ have been defined. Consider the least $i$ such that the antichain $U_{i}$ meets the interval $\left(t_{n-1}, t\right)$, and let $t_{n}$ be the (unique) point in $U_{i} \cap\left(t_{n-1}, t\right)$.

To show that we have indeed defined a $T$-graph in this way, we have to check that $t=\sup \left\{t_{n} \mid n \in \omega\right\}$. However this is clear, because the $t_{n}$ come from antichains $U_{i}$ with arbitrarily large $i$ : if $t \neq t^{\prime}:=\sup \left\{t_{n} \mid n \in \omega\right\}$ and 
$t^{\prime} \in U_{j}$, say, then every $t_{n}$ coming from an antichain $U_{i}$ with $i>j$ would have been chosen incorrectly.

To verify $(*)$, consider any $t^{\prime}>t$. This point $t^{\prime}$ has only finitely many neighbours below $t$, and the set of these neighbours depends only on the points in $\lceil t\rceil^{\circ}$ (more precisely, on which of the antichains $U_{i}$ these points belong to), not on the choice of $t^{\prime}$. We may therefore define $S_{t}$ as the set of those neighbours.

We have not investigated whether some or all of the Aronszajn-tree graphs we constructed in the proof of Theorem 6.2 are minor-minimal without a normal spanning tree (up to minor-equivalence). Moreover, we do not know whether every connected graph that has neither a normal spanning tree nor an overloaded minor contains one of them as a minor - in which case we could replace the arbitrary Aronszajn-tree graphs in Theorem 3.7 with these particular ones.

\section{Normal spanning trees and colouring number}

The colouring number of a graph $G$ is the least cardinal $\kappa$ such that $V(G)$ has a well-ordering in which each vertex is preceded by fewer than $\kappa$ of its neighbours (so that the greedy algorithm run on this ordering will use at most $\kappa$ colours). If $G$ has a normal spanning tree then clearly it has colouring number at most $\aleph_{0}$ : just well-order level by level.

Any $\left(\aleph_{0}, \aleph_{1}\right)$-graph $(A, B)$, on the other hand, has colouring number $\aleph_{1}$. Indeed, consider any well-ordering of its vertices, and let us find a vertex that is preceded by infinitely many of its neighbours. If every $b \in B$ is preceded by only finitely many of its neighbours, we may choose for each $b$ a neighbour $a=a(b)$ that appears later than $b$ in our well-ordering. As $B$ is uncountable but $A$ is countable, some $a \in A$ has the form $a=a(b)$ for uncountably many $b$, so $a$ is a vertex as desired.

Halin [9] conjectured that a connected graph has a normal spanning tree if and only if none of its minors has colouring number $\aleph_{1}$. (Note that while the property of admitting a normal spanning tree is closed under taking connected minors, the property of having countable colouring number is not. A complete graph, for example, has colouring number its own cardinal, while subdividing every edge once yields a graph of colouring number 3.) Since $\left(\aleph_{0}, \aleph_{1}\right)$-graphs have colouring number $\aleph_{1}$, this would indeed have followed from his (incorrect) conjecture that every connected graph without a normal spanning tree has an overloaded minor.

However, we can now prove Halin's colouring number conjecture from our Theorem 3.7:

Theorem 7.1. A connected graph has a normal spanning tree if and only if none of its minors has colouring number $\aleph_{1}$. 
Proof. The forward implication follows at once from the fact that graphs with normal spanning trees have countable colouring number and every component of a minor of a graph with a normal spanning tree also has a normal spanning tree (Cor. 1.2).

By Theorem 3.7 and our observation that $\left(\aleph_{0}, \aleph_{1}\right)$-graphs have colouring number $\aleph_{1}$, it thus suffices to prove for the converse implication that every $T$ graph $G$ with $T$ an Aronszajn tree has uncountable colouring number. ( $G$ trivially has colouring number at most $\aleph_{1}$, because $|T|=\aleph_{1}$.) Consider any wellordering of $T$; we have to find a vertex $t \in T$ that has infinitely many neighbours preceding it in this well-ordering.

If $T$ has a limit whose (infinitely many) lower neighbours in $T$ all precede it in our well-ordering, there is nothing more to show. So we may assume that every limit $x \in T$ has a lower neighbour $y=y(x)$ such that $x$ precedes $y$ in the well-ordering. For every non-zero limit ordinal $\alpha<\omega_{1}$ pick a vertex $x_{\alpha} \in T^{\alpha}$, let $y_{\alpha}:=y\left(x_{\alpha}\right)$, and let $f(\alpha)$ denote the unique limit ordinal $\beta<\alpha$ such that $y_{\alpha} \in T^{\beta+n}$ for some $n \in \omega$. Then $f$ is a (regressive) function from the set $L$ of non-zero limits in $\omega_{1}$ to $L \cup\{0\}$, and by our weak version of Fodor's lemma (2.1) there is an ordinal $\beta$ such that $f(\alpha)=\beta$ for every $\alpha$ in some uncountable set $U \subseteq L$. Then every $y_{\alpha}$ with $\alpha \in U$ lies at a level $T^{\beta+n}$ for some $n \in \omega$. As there are only countably many $n$ and each level $T^{\beta+n}$ is countable, there is even one vertex $t$ such that $t=y_{\alpha}=y\left(x_{\alpha}\right)$ for uncountably many $\alpha$. As each of the corresponding neighbours $x_{\alpha}$ of $t$ precedes $t$ in our well-ordering, $t$ is as desired.

As a curious spin-off, Theorem 7.1 implies that every graph of uncountable colouring number has a minor of colouring number exactly $\aleph_{1}$-a fact that does not appear to be obvious from the definitions. However, this is not a new result. Confirming and extending a conjecture of Milner, Komjáth [13] proved the much stronger result that, for every (finite or infinite) cardinal $\kappa \geqslant 2$, every graph of colouring number at least $\kappa$ has a subgraph of colouring number exactly $\kappa$.

\section{Classifying the $\left(\aleph_{0}, \aleph_{1}\right)$-graphs: an open problem}

Although we began to look at $\left(\aleph_{0}, \aleph_{1}\right)$-graphs in the course of our attempt to characterize the graphs admitting a normal spanning tree, their definition is so natural that the classification of these graphs seems to us to be a problem well worth studying in its own right. In this section, we present our preliminary findings: apart from the $\left(\aleph_{0}, \aleph_{1}\right)$-graphs of binary type defined in Section 3 (and a generalization of them defined below), we have found one other type of $\left(\aleph_{0}, \aleph_{1}\right)$-graph. We shall call those graphs indivisible $\left(\aleph_{0}, \aleph_{1}\right)$-graphs (for reasons soon to become clear), and we can show that they neither contain nor are contained in $\left(\aleph_{0}, \aleph_{1}\right)$-graphs of binary type as minors. 
Before we introduce these indivisible $\left(\aleph_{0}, \aleph_{1}\right)$-graphs, let us mention a generalization of $\left(\aleph_{0}, \aleph_{1}\right)$-graphs of binary type that may play a role in the classification of the $\left(\aleph_{0}, \aleph_{1}\right)$-graphs. The vertex set $T$ of these graphs is again any binary tree with $\aleph_{1}$ tops (see Section 3 ), and all their edges run between $T^{\omega}$ and $T^{<\omega}$. However, rather than joining every $t \in T^{\omega}$ to infinitely many points below it, we just require that its neighbourhood is a set of unbounded $\lceil t\rceil^{\circ}$-height in $T^{<\omega}$. These graphs are clearly again $\left(\aleph_{0}, \aleph_{1}\right)$-graphs, but they are considerably more general than those of binary type. (For example, it is not difficult to construct graphs of this kind that have a $K_{\aleph_{0}, \aleph_{1}}$ minor.) We do not know, however, whether every such graph contains an $\left(\aleph_{0}, \aleph_{1}\right)$-graph of binary type or an indivisible $\left(\aleph_{0}, \aleph_{1}\right)$-graph as a minor.

Let us now introduce our indivisible $\left(\aleph_{0}, \aleph_{1}\right)$-graphs. Our hope in constructing them was to find a kind of $\left(\aleph_{0}, \aleph_{1}\right)$-graph that differs essentially from those of binary type (which can be 'split in half', to yield two new $\left(\aleph_{0}, \aleph_{1}\right)$ graphs of binary type; see below). To make this idea more precise, we need some more definitions.

Let $(A, B)$ be an $\left(\aleph_{0}, \aleph_{1}\right)$-graph. Call a vertex $b \in B$ a strong neighbour of a set $A^{\prime} \subseteq A$ if it has infinitely many neighbours in $A^{\prime}$. A set $A^{\prime} \subseteq A$ is big if it has uncountably many strong neighbours. If a big set $A^{\prime}$ has two disjoint big subsets, we say that $A^{\prime}$ splits (into these subsets). Note that if $A_{1}, A_{2}$ are disjoint big sets, one can find disjoint subsets $B_{1}, B_{2}$ of $B$ such that $\left(A_{1}, B_{1}\right)$ and $\left(A_{2}, B_{2}\right)$ are both $\left(\aleph_{0}, \aleph_{1}\right)$-graphs. We call $(A, B)$ divisible if $A$ splits; otherwise $(A, B)$ is indivisible.

The following proposition shows that binary and indivisible $\left(\aleph_{0}, \aleph_{1}\right)$-graphs cannot be minors of each other:

Proposition 8.1. Let $(A, B) \succcurlyeq\left(A^{\prime}, B^{\prime}\right)$ be two $\left(\aleph_{0}, \aleph_{1}\right)$-graphs.

(i) If $(A, B)$ is of binary type then $\left(A^{\prime}, B^{\prime}\right)$ is divisible.

(ii) If $(A, B)$ is indivisible then $\left(A^{\prime}, B^{\prime}\right)$ is indivisible too (and hence not of binary type).

Proof. (i) Let $T$ be a binary tree with $\aleph_{1}$ tops such that $(A, B)$ is a $T$-graph with $A=T^{<\omega}$. We show that $A^{\prime}$ splits.

Let $X \subseteq B^{\prime}$ be the (uncountable) set of those vertices in $B^{\prime}$ whose branch set avoids $T^{<\omega}$. The branch sets of vertices in $X$ are singleton subsets of $T^{\omega}$, and we shall consider $X$ as a subset of $T^{\omega}$. Let $R$ be the set of $t \in T^{<\omega}$ with $\lfloor t\rfloor \cap X$ uncountable. Note that $R$ has at least one point at each level of $T^{<\omega}$.

If $R$ is a branch of $T^{<\omega}$, we delete all the points from $X$ that lie above any $t \notin R$. These are only countably many points, and the remaining uncountably many points of $X$ each lie above every point in $R$. But in $T^{\omega}$ there is only one such point, a contradiction.

So $R$ contains two incomparable points $t_{1}, t_{2}$. Delete the finitely many $a \in A^{\prime}$ whose branch set meets $\left\lceil t_{1}\right\rceil \cup\left\lceil t_{2}\right\rceil$. For each $i=1,2$, all the remaining 
neighbours of $B_{i}^{\prime}:=\left\lfloor t_{i}\right\rfloor \cap X$ in $A^{\prime}$ have their branch sets in $\left\lfloor t_{i}\right\rfloor$. These sets $N\left(B_{1}^{\prime}\right)$ and $N\left(B_{2}^{\prime}\right)$ are big disjoint subsets of $A^{\prime}$, as desired.

(ii) We assume that $A^{\prime}$ splits into big sets $A_{1}^{\prime}$ and $A_{2}^{\prime}$, and show that $A$ splits, too. For each $i=1,2$, uncountably many of the strong neighbours of $A_{i}^{\prime}$ in $B^{\prime}$ have branch sets avoiding $A$, so these branch sets are singleton elements of $B$. Each of them sends infinitely many edges of $(A, B)$ to

$$
A_{i}:=A \cap \bigcup_{a \in A_{i}^{\prime}} X_{a}
$$

where $X_{a}$ denotes the branch set of $a$. Hence $A_{1}$ and $A_{2}$ are big in $(A, B)$, and $A$ splits into these two sets.

Having thus shown why indivisible $\left(\aleph_{0}, \aleph_{1}\right)$-graphs are interesting, let us not forget to prove their existence:

Proposition 8.2. If $\mathrm{CH}$ holds, then there exists an indivisible $\left(\aleph_{0}, \aleph_{1}\right)$-graph.

Proof. Let $A$ and $B$ be sets of cardinality $\aleph_{0}$ and $\aleph_{1}$, respectively. Our task is to define edges between $A$ and $B$ so that $(A, B)$ becomes an indivisible $\left(\aleph_{0}, \aleph_{1}\right)$-graph.

Let $\mathcal{U}$ be a non-principal ultrafilter on $A$. Let $\left(A_{\alpha}\right)_{\alpha<\omega_{1}}$ be a well-ordering of the subsets of $A$ that are not in $\mathcal{U}$, and let $\left(b_{\alpha}\right)_{\alpha<\omega_{1}}$ be a well-ordering of $B$. Our aim is to choose the edges of $(A, B)$ so that

For all $\alpha<\beta<\omega_{1}$, the vertex $b_{\beta}$ is adjacent to only finitely many vertices in $A_{\alpha}$.

Then each of the sets $A_{\alpha}$ will have only countably many strong neighbours, so every big subset of $A$ will be in $\mathcal{U}$. Since $\mathcal{U}$ does not contain two disjoint sets, $A$ will not split.

So let us choose the edges at $b_{\beta}$ so as to satisfy $(*)$. Enumerate $\left\{A_{\alpha} \mid \alpha<\beta\right\}$ as $A^{1}, A^{2}, \ldots$, choose distinct vertices $a_{i} \in A \backslash\left(A^{1} \cup \ldots \cup A^{i}\right)$ for all $i \in \mathbb{N}$ (using that $\mathcal{U}$ is non-principal), and make $b_{\beta}$ adjacent to these vertices $a_{1}, a_{2}, \ldots$.

We remark that $\mathrm{CH}$ is indeed needed for Proposition 8.2 to be true: if $\mathrm{CH}$ fails and Martin's Axiom MA $\left(\omega_{1}\right)$ holds, then there is no indivisible $\left(\aleph_{0}, \aleph_{1}\right)$ graph (J.M. Brochet, personal communication).

Despite the seemingly far-fetched construction in the proof of Proposition 8.2, the indivisible $\left(\aleph_{0}, \aleph_{1}\right)$-graphs constructed there are quite canonical in that every indivisible $\left(\aleph_{0}, \aleph_{1}\right)$-graph $(A, B)$ has a subgraph of that kind. Indeed, it is easily checked that of any pair of complementary subsets of $A$ exactly one is big and these big sets form a non-principal ultrafilter on $A$. Thus if $\left(A_{\alpha}\right)_{\alpha<\omega_{1}}$ is a well-ordering of the subsets of $A$ that are not big, we 
may inductively for all $\beta<\omega_{1}$ choose $b_{\beta} \in B$ from outside the (countably many countable) sets of strong neighbours of the $A_{\alpha}$ with $\alpha<\beta$. These $b_{\beta}$ then satisfy condition $(*)$ in the proof of Proposition 8.2.

We do not know whether indivisible $\left(\aleph_{0}, \aleph_{1}\right)$-graphs giving rise to the same ultrafilter in this way are necessarily minors of each other. Moreover, we do not know whether the two types of $\left(\aleph_{0}, \aleph_{1}\right)$-graph we have seen in this section are essentially all:

Problem. Does every $\left(\aleph_{0}, \aleph_{1}\right)$-graph have an $\left(\aleph_{0}, \aleph_{1}\right)$-graph minor that is either indivisible or of binary type?

We suspect the answer to this question to be 'no', but have been unable to construct a counterexample.

\section{A new antichain of infinite graphs}

By the Robertson-Seymour Graph Minor Theorem (see [4]), there is no infinite set of finite graphs none of which is a minor of any other. Thomas [15] constructed an infinite sequence of uncountable graphs such that no graph in this sequence is a minor of a later one (hence showing that the uncountable graphs are not well-quasi-ordered by the minor relation), i.e. a sequence containing either an infinite minor-antichain or an infinite descending chain. (Incidentally, Thomas's graphs are all binary trees with tops.) Komjáth [12] constructed a minor-antichain of size $2^{\kappa}$ of graphs of order $\kappa$, for every uncountable ordinal $\kappa$.

The purpose of this section is to observe that the indivisible $\left(\aleph_{0}, \aleph_{1}\right)$-graphs discussed in Section 8 form minor-antichains of size $2^{2^{\aleph_{0}}}$. So we reobtain the first case of Komjáth's result, yet with a more transparent construction.

Let $(A, B) \succcurlyeq\left(A^{\prime}, B^{\prime}\right)$ be indivisible $\left(\aleph_{0}, \aleph_{1}\right)$-graphs. Let $\mathcal{U}$ and $\mathcal{U}^{\prime}$ be the corresponding ultrafilters of big subsets of $A$ and $A^{\prime}$, respectively. For every $a^{\prime} \in A^{\prime}$, let $A_{a^{\prime}}$ be the intersection of the branch set of $a^{\prime}$ with $A$. It is now easy to check that, for every $A^{\prime \prime} \subseteq A^{\prime}$, the set

$$
\bigcup_{a^{\prime} \in A^{\prime \prime}} A_{a^{\prime}} \subseteq A
$$

is big in $(A, B)$ if and only if $A^{\prime \prime}$ is big in $\left(A^{\prime}, B^{\prime}\right)$. But this means that $\mathcal{U} \geqslant \mathcal{U}^{\prime}$ in the Rudin-Keisler pre-order on the ultrafilters on a countable set (see [2]). But for any set of fewer than $2^{2^{\aleph_{0}}}$ non-principal such ultrafilters there is another that is incomparable with them all in this pre-order, so we can inductively find an antichain of size $2^{2^{\aleph_{0}}}$. By the construction of Proposition 8.2 these ultrafilters correspond to indivisible $\left(\aleph_{0}, \aleph_{1}\right)$-graphs, which are then minor-incomparable by the observation above. 
Acknowledgement. We thank Stevo Todorčević for pointing out that special Aronszajn trees can be used canonically in the proof of Theorem 6.2 to establish our desired property $(*)$. This not only replaces our earlier more long-winded direct construction of an Aronszajn tree $T$ together with a $T$-graph with $(*)$, but also shows that Aronszajn-tree graphs without overloaded minors are rather more common than we had thought.

\section{References}

[1] J. M. Brochet and R. Diestel, Normal tree-orders for infinite graphs, Trans. Amer. Math. Soc. 345 (1994), 871-895.

[2] W.W. Comfort and S. Negrepontis, The Theory of Ultrafilters, Springer-Verlag 1974.

[3] R. Diestel, Graph decompositions: a study in infinite graph theory, Oxford University Press, Oxford 1990.

[4] R. Diestel, Graph theory, 2nd edition, Springer-Verlag 2000 and http://www.math. uni-hamburg.de/home/diestel/books/graph.theory/download.html

[5] R. Diestel, The structure of TK $K_{a}$-free graphs, J. Combin. Theory B 54 (1992), 222-238.

[6] R. Diestel, The depth-first search tree structure of $\mathrm{T} K_{\aleph_{0}}$-free graphs, J. Combin. Theory B 61 (1994), 260-262.

[7] R. Diestel, The classification of finitely spreading graphs, Proc. London Math. Soc. (3) 73 (1996), 534-554.

[8] R. Diestel and I. Leader, A proof of the bounded graph conjecture, Invent. math. 108 (1992), 131-162.

[9] R. Halin, Miscellaneous problems on infinite graphs, J. Graph Theory (to appear).

[10] T. Jech, Set Theory, Academic Press 1978.

[11] H. A. Jung, Wurzelbäume und unendliche Wege in Graphen, Math. Nachr. 41 (1969), $1-22$.

[12] P. Komjáth, A note on minors of uncountable graphs, Math. Proc. Camb. Phil. Soc. 117 (1995), 7-9.

[13] P. Komjáth, Two remarks on the coloring number, J. Combin. Theory B 70 (1997), 301-305.

[14] D. Kurepa, Ensembles Ordonnés et Ramifiés, Publ. Math. Univ. Belgrade 4 (1935), 1138.

[15] R. Thomas, A counter-example to 'Wagner's conjecture' for infinite graphs, Math. Proc. Camb. Phil. Soc. 103 (1988), 55-57.

Reinhard Diestel

Mathematisches Seminar

Universität Hamburg

Bundesstraße 55

D - 20146 Hamburg

Germany
Imre Leader

Department of Mathematics

University College London

Gower Street

GB - London WC1E 6BT

England 